\title{
Tracking of Proton Transfer Reaction in Supercooled RNA Nucleoside
}

\author{
Z. Wojnarowska, ${ }^{* \dagger}$ M. Paluch, ${ }^{\dagger}$ P. Wlodarczyk, ${ }^{\dagger}$ M. Dulski, ${ }^{\dagger}$ R. Wrzalik, ${ }^{\dagger}$ and C. M. Roland ${ }^{\ddagger}$ \\ ${ }^{\dagger}$ Institute of Physics, University of Silesia, ul. Uniwersytecka 4, 40-007 Katowice, Poland \\ ${ }^{\ddagger}$ Naval Research Laboratory, Chemistry Division, Code 6120, Washington, DC 20375-5342, United States
}

ABSTRACT: In this paper we address a longstanding debate concerning the proton transfer reaction of DNA and RNA compounds. The sample tested is $\beta$ uridine, one of the four basic components of RNA, which has been suggested to have the capacity to adopt lactam and lactim tautomeric forms. The essential biological importance of this RNA nucleoside has motivated a number of experimental and theoretical investigations in the past. However, the kinetics of the proton transfer reaction of this compound has not been examined. Herein, we employ dielectric spectroscopy and refractive index measurements to experimentally characterize lactam-lactim transformation in the supercooled liquid state. We quantify the kinetics of $\beta$-uridine tautomerization and suggest a mechanism for the configurational changes. The obtained data are compared to quantum chemical calculations. Finally the biological importance of our results is discussed.

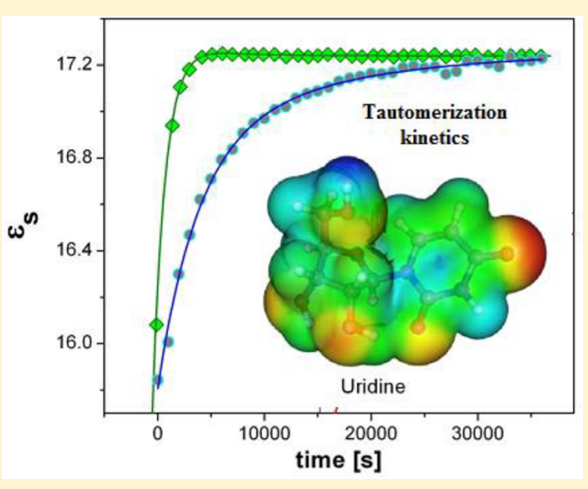

SECTION: Biophysical Chemistry and Biomolecules

$\mathrm{T}$ automerization, the reversible proton migration in an organic molecule, ${ }^{1}$ is of great importance in physics, chemistry, molecular biology, pharmacology, and so forth because of its occurrence in various organic compounds, including saccharides (ring-chain tautomerization comprising a well-known mutarotation process ${ }^{2,3}$ ), pharmaceuticals (e.g., amide-imidic acid transformation of antidiabetic drugs ${ }^{4,5}$ ), and biomaterials or other chemical substances. ${ }^{6}$ An exciting issue in molecular biology is tautomerization of compounds forming the nucleic acids DNA and RNA. ${ }^{7,8}$ Since the investigations of Watson and Crick, it is known that five nucleic acids and bases can exist in two or more isomeric forms, differing only in the location of a single proton. ${ }^{9,10}$ While the tautomers of purines (adenine $\mathrm{A}$ and guanine $\mathrm{G}$ ) have different positions of the hydrogen around the base, in the case of pirymidines (cytosine $\mathrm{C}$, thymine $\mathrm{T}$, and uracil $\mathrm{U}$ ) the lactam-lactim tautomerism consists of an amide-imidic acid transformation in heterocyclic rings. ${ }^{11}$ A large amount of research has demonstrated that the existence of nucleobases in a canonic structure is essential for storage and transduction of hereditary information. ${ }^{12}$ Genetic information contained in the base sequence must be accurately sustained through several processes, including DNA replication, transcription, DNA repair, recombination, translation, and RNA interference. Therefore, if one nucleic base is replaced by its tautomer, incorrect base pairing can result, introducing errors in the genetic code, i.e., a mutation. ${ }^{13}$ The result can be medically significant, such as genetic disease, cancer, or drug resistance. $^{14}$

However, the assessment of the significance of point mutation processes requires knowledge of the kinetics of proton transfer reactions in nucleobases, that is, the rate constant and activation energy barrier. ${ }^{15}$ At the present time, such information comes mainly from ab initio theoretical calculations of tautomerization in isolated or hydrated molecules. $^{16}$ Experimental verification of these theoretical predictions is lacking, due to the absence of a suitable experimental technique to detect a small amount of noncanonic RNA and DNA tautomers, as required for the determination of the kinetic parameters of the proton transfer reactions. ${ }^{15} \mathrm{We}$ demonstrate herein the application of broadband dielectric spectroscopy (BDS), traditionally used to measure the molecular dynamics of liquids and glasses, ${ }^{17}$ to tracking the tautomerization process of an RNA compound. We have determined both the activation energy barrier and the time scales of proton transfer reaction of the nucleoside, $\beta$-uridine. These kinetic results are compared to refractive index measurements; quantum chemical calculations were also performed. The biological consequences of this process are discussed.

Herein, we have investigated the lactam-lactim tautomerism of anhydrous $\beta$-uridine (Figure 1). The crystalline sample (99\% purity) was purchased from Sigma Aldrich and used as received. Dielectric spectroscopy employed a Novo-Control GMBH Alpha dielectric spectrometer, with measurements in the frequency range from $10^{-1} \mathrm{~Hz}$ to $10^{6} \mathrm{kHz}$ at temperatures from 342 to $363 \mathrm{~K}$. Temperature was controlled by a Quatro system, employing a nitrogen-gas cryostat with temperature stability better than $0.1 \mathrm{~K} / \mathrm{s}$. As a complementary experimental technique, UV-vis spectroscopy (AFS2000, OceanOptics) was used to monitor the time evolution of the refractive index at various temperatures, employing the procedure described in refs 18 and 19.

Received: May 1, 2012

Accepted: August 1, 2012

Published: August 2, 2012 


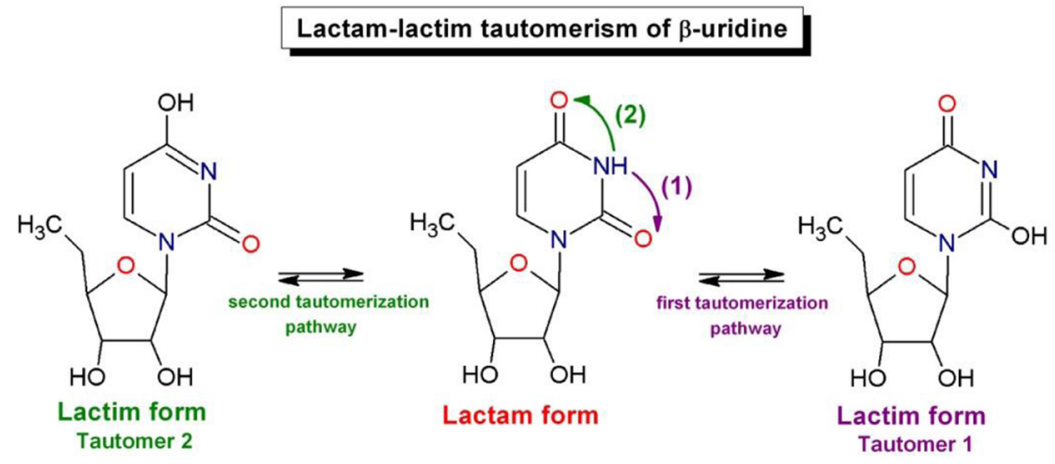

Figure 1. $\beta$-Uridine and two possible noncanonical tautomeric forms.

Since the tautomerization process is possible only for certain conditions, i.e., in solution, the gas phase, or in the amorphous liquid state, $\beta$-uridine was prepared by supercooling below its melting point $(=438 \pm 2 \mathrm{~K})$. Dielectric spectra (real, $\varepsilon^{\prime}$, and imaginary, $\varepsilon^{\prime \prime}$, permittivity) are displayed in the upper panel of Figure 2 for the amorphous material. These were obtained for
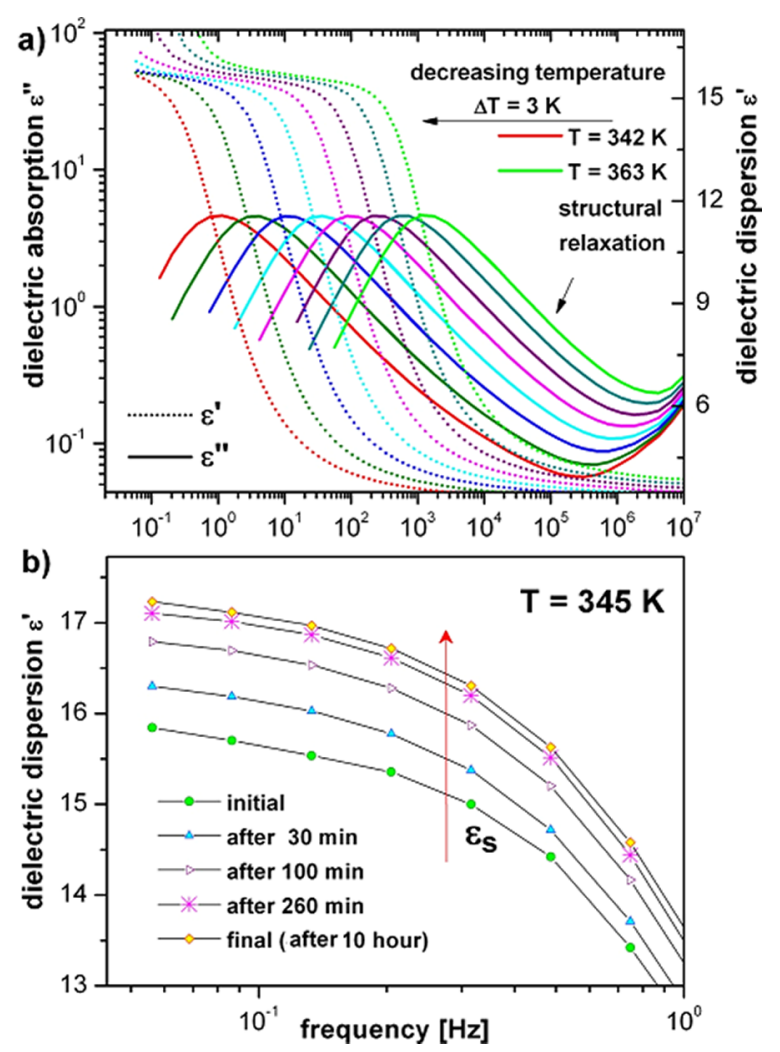

Figure 2. (a) Frequency variation of the real $\left(\varepsilon^{\prime}\right)$, and imaginary $\left(\varepsilon^{\prime \prime}\right)$, components of the permittivity for $\beta$-uridine at ambient pressure and temperatures in the range of $342-363 \mathrm{~K}$ in steps of $3 \mathrm{~K}$. The contribution of direct current $(\mathrm{dc})$ conductivity to $\varepsilon^{\prime \prime}$ has been subtracted from the loss spectra. (b) Changes in the real part of the permittivity during the isothermal annealing at $345 \mathrm{~K}$.

various temperatures during heating from 342 to $363 \mathrm{~K}$. The solid lines indicate the resolved dielectric loss peak, referred to as the structural $(\alpha)$ relaxation process, which moves toward higher frequencies with increasing temperature. This process originates from the cooperative motions of the molecule and is related to the glass transition of $\beta$-uridine. ${ }^{20}$ Defined as the temperature at which the structural relaxation time $\left(\tau_{\alpha}=1\right.$ /
$2 \pi f_{\max }$ ) equals $100 \mathrm{~s}$, we determine the glass transition temperature, $T_{g}$, to equal $324 \mathrm{~K}$. The $\varepsilon^{\prime}(f)$ in Figure 2a exhibits a characteristic step-change in behavior, with the lowfrequency limiting value, the static permittivity $\varepsilon_{\text {s }}$, governed by the average dipole moment $\left(\mu^{2}\right)$ and the number of dipoles $(N):^{21,22}$

$$
\Delta \varepsilon=\varepsilon_{s}-\varepsilon_{\infty} \approx N \cdot \mu^{2}
$$

The tautomerization process will change $\varepsilon_{\mathrm{s}}$ if the dipole moments of the isomers have different magnitudes. ${ }^{23}$ Thus, the isothermal static permittivity as a function of time reflects the kinetics of the lactam-lactim tautomerization of $\beta$-uridine. At $345 \mathrm{~K}$, which is close to $T_{\mathrm{g}}$, tautomerization will be sufficiently slow to be readily monitored. As illustrated in Figure $2 \mathrm{~b}$, over 10 hours at $345 \mathrm{~K}, \varepsilon_{\mathrm{s}}$ increased almost $9 \%$ from its initial value. Note that over this same time, the structural relaxation process did not change, meaning that the viscosity was constant. The same pattern of behavior, reflecting lactam-lactim izomerization, was also observed at higher temperatures, 348 and $351 \mathrm{~K}$. However, above $351 \mathrm{~K}$, equilibrium was achieved too fast to allow the proton transfer to be monitored.

To establish the rate of uridine isomerization, the evolution in time of the static permittivity at each temperature was analyzed, with representative $\varepsilon_{\mathrm{s}}(t)$ for 345 and $351 \mathrm{~K}$ shown in Figure 3. Initially the assumption of first-order kinetics was made; however, a single exponential time-dependence cannot describe the experimental data satisfactorily. A very good fit was achieved using a superposition of two first-order kinetic equations:

$$
\varepsilon_{\mathrm{s}}=A_{1} \cdot \exp \left(-k_{1} \cdot t\right)+A_{2} \cdot \exp \left(-k_{2} \cdot t\right)+C
$$

Consequently, values for two rate constants, $k_{1}$ and $k_{2}$, were determined for each temperature. This analysis reveals that during the isothermal equilibration, two different tautomeric conversions of the uridine occur simultaneously. Both rate constants exhibit Arrhenius behavior (Figure 3b), with activation energy barriers for each tautomeric pathway in supercooled uridine equal to $E_{\mathrm{a} 1}=188 \pm 15 \mathrm{~kJ} / \mathrm{mol}$ and $E_{\mathrm{a} 2}=$ $215 \pm 3 \mathrm{~kJ} / \mathrm{mol}$.

To correctly assign these activation energies to the appropriate tautomeric conversions, the energy barriers of lactam-lactim tautomerizm were estimated theoretically. This requires identifying the mechanisms of tautomerization; that is, does proton transfer during the uridine equilibration involve the lactam-lactim conversion or its opposite? Experimentally, we find (Figure 2a) that the average dipole moment increases with time at constant temperature. This means that the population of isomers having larger a dipole moment increases 

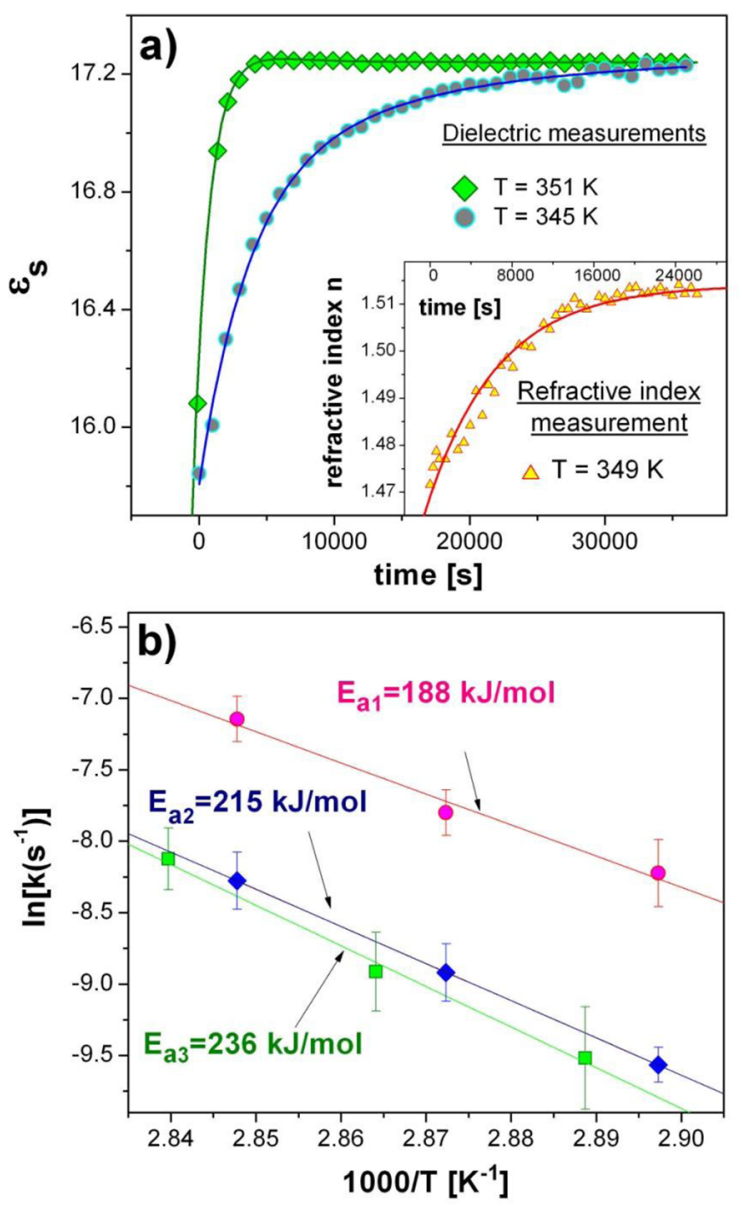

Figure 3. (a) Static permittivity of $\beta$-uridine during annealing at 345 and $351 \mathrm{~K} . \varepsilon_{\mathrm{s}}$ was determined at 0.055 and $0.3 \mathrm{~Hz}$ for 345 and $351 \mathrm{~K}$, respectively. The inset shows the time variation of the refractive index. (b) Arrhenius plots of the rate constants from dielectric (circles and diamonds) and refractive index (squares) measurements, with the activation energies indicated. Solid lines are fits.

during chemical equilibration. To calculate the dipole moments of the possible tautomeric isomers of uridine, we employed density functional theory (DFT), a popular quantum mechanical computational method. The specified directions and values of the dipole moments for all tautomers and their transition state (TS) are depicted in Figure 4. As can be seen, for both noncanonical tautomeric structures (Tautomers 1 and 2), the dipole moments are higher than that calculated for the lactam form. This indicates that conversion of lactam to both lactims transpires during the time dependent dielectric measurements. The tautomers of uridine lactam are the most energetically stable, with high temperature shifting the equilibrium toward the less stable tautomeric species. If the opposite reaction took place, a decrease of the static permittivity would be observed.

Next we applied computational methods to obtain the activation energies of both tautomerization pathways of uridine. As illustrated in Figure 4, the values of $E_{\mathrm{a}}$ determined using DFT model B3LYP/6-311++G(d,p) equal 182 and $216 \mathrm{~kJ} /$ mol, in fair agreement with the experimental results. The activation energy of proton transfer reaction leading to the lactim form (designated "Tautomer 2" in Figure 4), is $34 \mathrm{~kJ} /$ mol lower than the alternate reaction; thus, the probability of formation of Tautomer 2 is higher. The following question arises: What might be the biological consequences of this conversion? To shed light on this, we calculated the electrostatic potential on the surfaces of lactam and two possible lactim tautomers of uridine. Since in the WatsonCrick model of the RNA helix uridine is complementary to adenosine, the electrostatic potential of the latter was also evaluated. As seen in Figure 5, the uridine isomers have different electrostatic potentials on their molecular surfaces, which implies different $\mathrm{H}$-bonds formation abilities; i.e., the site formerly a hydrogen donor becomes a proton acceptor. On the other hand, Tautomer 2 is very similar to adenosine, and therefore can mimic this nucleoside. Such a nucleoside replacement can have serious biological consequences, because ribonucleic acid, in which uridine is naturally occurring, is essential to life.

RNA has an active role in protein synthesis. In this "translation" mechanism, three types of RNA participate: messenger RNA (mRNA), which carries the genetic information copied from DNA; transfer RNA (tRNA), involved in deciphering the mRNA code, and ribosomal RNA (rRNA), associated with a set of proteins that form ribosomes. Each molecule of tRNA transports its own amino acid, and contains three-base sequences that can be paired with its complementary code in mRNA. The information in mRNA must be properly read by tRNA for the suitable peptide chain to be constructed. For example, the sequence of UCC in mRNA is complementary to code AGG in tRNA, and, consequently, serine is attached to the amino acid chain. ${ }^{24}$ However, if the tautomerization process occurs, uridine tautomers may be interpreted as adenosine molecules. In such a situation, the UCC sequence in mRNA may be recognized as ACC, and consequently the amino acid chain trypsin incorporated rather than serine. Accumulation of abnormally constructed proteins leads to cell damage, and potentially diseases such as Alzheimer's or Parkinson's. ${ }^{25,26}$

The relevant question becomes what is the rate of proton transfer in uridine at biological temperatures. To assess this, the fits of the Arrhenius equation to the temperature dependences of the rate constants (Figure $3 \mathrm{~b}$ ) were extrapolated to human body temperature; the respective values for $k_{1}$ and $k_{2}$ at $310 \mathrm{~K}$ were found to be $1.7 \times 10^{-8}$ and $2.5 \times 10^{-7} \mathrm{~s}^{-1}$. These correspond to equilibration times for proton transfer of about $14200 \pm 1100$ and $2800 \pm 100 \mathrm{~h}$, for the first and second tautomerization pathways, respectively. Similar results have been obtained theoretically for guanine tautomerization $(2500$ $\mathrm{h})$, which also involves lactam-lactim transformation. ${ }^{27}$ Although the reaction is slow, it cannot be ruled out as a source of point mutations or improper protein synthesis.

Finally, we monitored the tautomerization process by determining the refractive index of uridine as a function of time at various temperatures. Similarly to the dielectric spectroscopy results, we constructed kinetic curves for each measured temperature. The representative kinetic curve is depicted in the inset to Figure 3a. Interestingly, the first order kinetic equation was sufficient to describe the time dependence of the refractive index. As depicted in the inset to Figure $3 \mathrm{~b}$, only an average value of the activation energy of the tautomerization reaction is obtained, $E_{\mathrm{a} 3}=236 \pm 18 \mathrm{~kJ} / \mathrm{mol}$. This optical technique cannot distinguish between the tautomerization pathways, probably because the polarizabilities of the $\beta$-uridine tautomers are not very different.

In summary, we have demonstrated reversible proton migration in $\beta$-uridine, a component of RNA. The reaction 


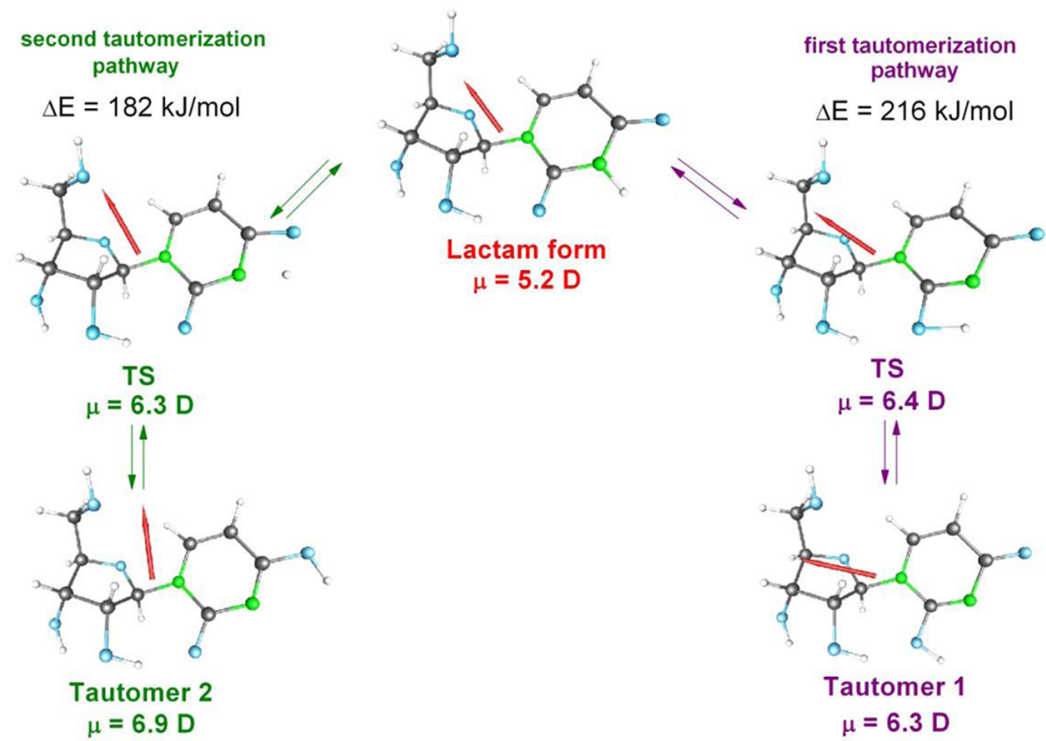

Figure 4. Dipole moment of possible $\beta$-uridine tautomers. Uridine geometries were determined using the orca package at the $\mathrm{B} 3 \mathrm{LYP} / 6-31+\mathrm{G}(\mathrm{d}, \mathrm{p})$ level. Energies and dipole moments were calculated at the B3LYP $/ 6-311++\mathrm{G}(\mathrm{d}, \mathrm{p})$ level within DFT $\left(E_{\mathrm{a} 1}=182 \mathrm{~kJ} / \mathrm{mol}, E_{\mathrm{a} 2}=216 \mathrm{~kJ} / \mathrm{mol}\right)$ and in the Dunning's cc-pvdz basis set within the coupled cluster method $\operatorname{CCSD}(\mathrm{T})$ in orca using the RI approximation $\left(E_{\mathrm{a} 1}=178 \mathrm{~kJ} / \mathrm{mol}, E_{\mathrm{a} 2}=208 \mathrm{~kJ} / \mathrm{mol}\right)$. First-order saddle points, necessary for computing reaction energy barriers, were optimized in orca at the B3LYP/6-31+G(d,p) level. Frequency analysis was performed to verify the order of saddle points and to calculate the zero point energy correction.

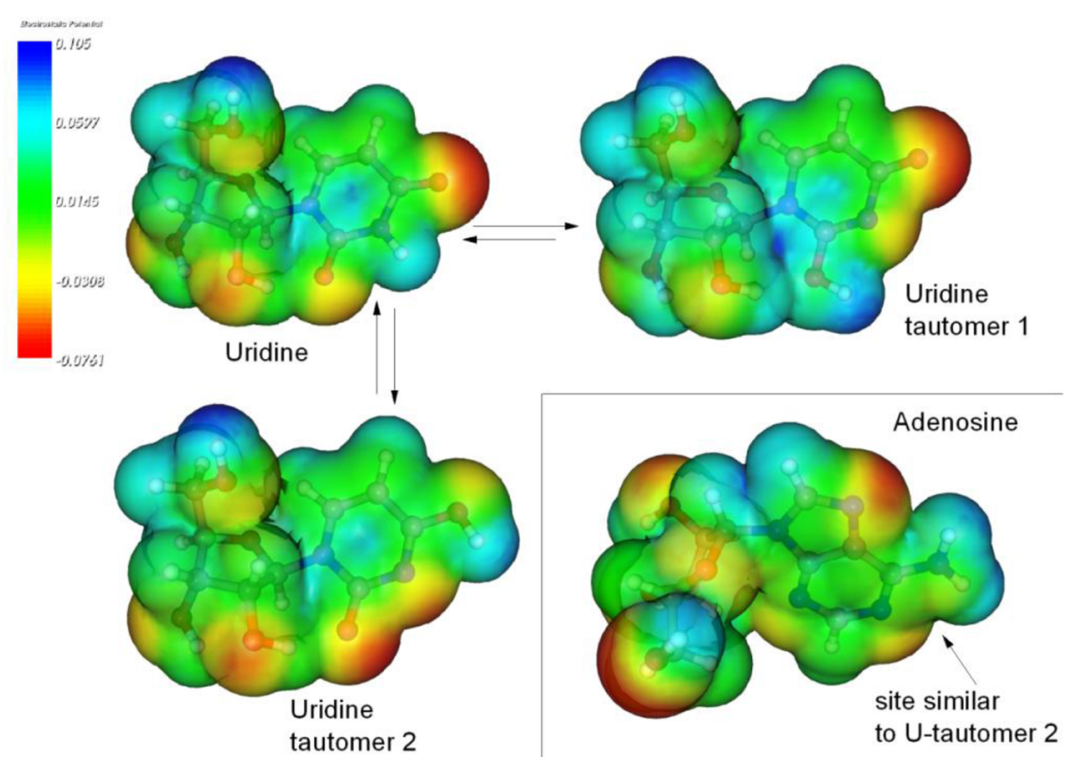

Figure 5. Electrostatic potential of $\beta$-uridine nucleoside (lactam form) and its two possible lactim tautomers. For comparison, the adenosine molecule and its electrostatic potential are shown. All potential surfaces were computed using the Gaussian 03 package.

involves two simultaneous conversions giving rise to two lactim tautomers, which have different dipole moments and different electrostatic surface potentials. We have shown that dielectric measurements make possible the monitoring of the kinetics of both tautomerization reactions. Since the kinetic factors determined experimentally are in good agreement with the computational results, the BDS technique offers a new approach to the study of proton transfer reactions of RNA and DNA compounds, and consequently for investigations of possible point mutations.

\section{AUTHOR INFORMATION}

\section{Corresponding Author}

*E-mail: zwojnaro@us.edu.pl.

\section{Notes}

The authors declare no competing financial interest.

\section{ACKNOWLEDGMENTS}

The work at NRL was supported by the Office of Naval Research. Z.W. and M.P. are deeply thankful for the financial support received from the National Science Center (Grant MAESTRO2).

\section{REFERENCES}

(1) Smith, M. B.; March, J. Advanced Organic Chemistry, 5th ed; Wiley Interscience: New York, 2001; pp 1218-1223.

(2) Carey, F. Organic Chemistry, 4th ed.; McGraw-Hill Higher Education Press: New York, 2000. 
(3) Wlodarczyk, P.; Kaminski, K.; Paluch, M.; Ziolo, J. Mutarotation in D-Fructose Melt Monitored by Dielectric Spectroscopy. J. Phys. Chem. B 2009, 113, 4379-4383.

(4) Wojnarowska, Z.; Grzybowska, K.; Adrjanowicz, K.; Kaminski, K.; Paluch, M; Hawelek, L.; Wrzalik, R.; Dulski, M.; Sawicki, W.; Mazgalski, J.; et al. Study of the Amorphous Glibenclamide Drug: Analysis of the Molecular Dynamics of Quenched and Cryomilled Material. Mol. Pharmaceutics 2010, 7, 1692-1707.

(5) Wojnarowska, Z.; Wlodarczyk, P.; Kaminski, K.; Grzybowska, K.; Hawelek, L.; Paluch, M. On the Kinetics of Tautomerism in Drugs New Application of Broadband Dielectric Spectroscopy. J. Chem. Phys. 2010, 133, 094507.

(6) Yvonne, M. Overview of the Perspectives Devoted to Tautomerism in Molecular Design. J. Comput.-Aided Mol. Des. 2010, 24 (6-7), 473-474.

(7) Goodman, M. F. Mutations Caught in the Act. Nature 1995, 378, 237-238.

(8) Topal, M. D.; Fresco, J. R. Complementary Base Pairing and the Origin of Substitution Mutations. Nature 1976, 263, 285-287.

(9) Watson, J. D.; Crick, F. H. C. Genetical Implications of the Structure of Deoxyribonucleic Acid. Nature 1953, 171, 964-967.

(10) Dreyfus, M.; Bensaude, O.; Dodin, G.; Dubois, J. E. Tautomerism in Cytosine and 3-Methylcytosine. A Thermodynamic and Kinetic Study. J. Am. Chem. Soc. 1976, 98, 6338-6349.

(11) Shugar, D.; Kierdaszuk, B. New Light on Tautomerism of Purines and Pyrimidines and Its Biological and Genetic Implications. Proc. Int. Symp. Biomol. Struct. Interact., Suppl. J. Biosci. 1985, 8, 657668.

(12) Saenger, W. Principles of Nucleic Acid Structure; Springer-Verlag: New York, 1984.

(13) Holbrook, S. R.; Cheong, Kim, S. H. Crystal Structure of an RNA Double Helix Incorporating a Track of Non-Watson-Crick Base Pairs. Nature 1991, 353, 579.

(14) Krueger, A. T.; Kool, E. T. Model Systems for Understanding DNA Base Pairing. Curr. Opin. Chem. Biol. 2007, 11, 588-594.

(15) Paneth, P., Kinetics and Dynamics: From Nano- to Bio-Scale; Springer: New York, 2010.

(16) Ha, T. K.; Keller, H. J.; Gunde, R.; Gunthard, H. H. Energy Increment Method Based on Quantum Chemical Results: A General Recipe for Approximative Prediction of Isomerization and Tautomerization Energies of Pyrimidine and Purine Nucleic Acid Bases and Related Compounds. J. Phys. Chem. A 1999, 103, 6612-6623.

(17) Lunkenheimer, P.; Schneider, U.; Brand, R.; Loidl, A. Glassy Dynamics. Contemp. Phys. 2000, 41, 15.

(18) Stuart, B.; George, B.; McIntyre, P. Modern Infrared Spectroscopy; John Wiley \& Sons: New York, 1998.

(19) Kossivas, F.; Kyprianou, A. Measurement of liquid crystal film thickness using interferometry. Meas. Sci. Technol. 2010, 21, 105707.

(20) Paluch, M.; Roland, C. M.; Pawlus, S.; Zioło, J.; Ngai, K. L. Does the Arrhenius Temperature Dependence of the Johari-Goldstein Relaxation Persist above $T_{\mathrm{g}}$ ? Phys. Rev. Lett. 2003, 91, 115701.

(21) Hill, N. E. The Application of Onsager's Theory to Dielectric Dispersion. Proc. Phys. Soc. 1958, 72, 532.

(22) Kremer, K.; Schonhals, A. Broadband Dielectric Spectroscopy; Springer: New York, 2002.

(23) Wlodarczyk, P.; Kaminski, K.; Haracz, S.; Dulski, M.; Paluch, M. Kinetic Processes in Supercooled Monosaccharides upon Melting: Application of Dielectric Spectroscopy in the Mutarotation Studies of D-Ribose. J. Chem. Phys. 2010, 132, 195104.

(24) Griffiths, A. Introduction to Genetic Analysis, 9th ed.; W.H. Freeman and Company: New York, 2008; pp 335-339. ISBN 978-07167-6887-6.

(25) Reynaud, E. Protein Misfolding and Degenerative Diseases. Nat. Educ. 2010, 3, 28.

(26) Dobson, C. M. Protein Misfolding Diseases: Getting out of Shape. Nature 2002, 418, 729-730.

(27) Gorb, L.; Kaczmarek, A.; Gorb, A.; Sadlej, A. J.; Leszczynski, J. Thermodynamics and Kinetics of Intramolecular Proton Transfer in
Guanine. Post Hartree-Fock Study. J. Phys. Chem. B 2005, 109, 13770-13776. 